\title{
A Compact Differential-Mode Wide Stopband Bandpass Filter with Good and Wideband Common-Mode Suppression
}

\author{
Gang Zhang $\mathbb{D}^{\mathbb{D}}$, Yang Zhao $\mathbb{D}^{\mathrm{D}}$, and Wei Yan \\ School of Electrical and Automation Engineering, Nanjing Normal University, Nanjing 210046, China \\ Correspondence should be addressed to Yang Zhao; yang_zhao_nnu@126.com
}

Received 21 December 2017; Accepted 18 February 2018; Published 21 March 2018

Academic Editor: Lei Ge

Copyright (c) 2018 Gang Zhang et al. This is an open access article distributed under the Creative Commons Attribution License, which permits unrestricted use, distribution, and reproduction in any medium, provided the original work is properly cited.

\begin{abstract}
This letter presents a microstrip differential-mode (DM) bandpass filter (BPF) with high and wide stopband suppression under both DM and common-mode (CM) operations. A new coupling topology is formed up to realize the DM BPF by integrating a pair of $\lambda / 2$ microstrip transmission lines and two multimode resonators. The DM and CM equivalent half-circuits are established to explain the operating principal. For validation, a DM BPF operating at $2.2 \mathrm{GHz}$ is implemented. Experimental results indicate that the presented DM BPF exhibits not only sharp DM passband selectivity but also deep and ultrawide stopband suppression of more than $30 \mathrm{~dB}$ for both DM and CM.
\end{abstract}

\section{Introduction}

Recently, the great development of modern wireless communication system has put forward higher requirements on $\mathrm{RF}$ devices with high performance and miniaturized size. To meet these requirements, one effective approach is to design multifunction embedded component by integrating multiple functions into one component. Since differential-mode circuits play an important role in the modern communication system as they can reduce the environmental noise as well as crosstalk in the system and bandpass filters (BPFs) can realize the passband frequency selective response, differential-mode bandpass filter as one typical integrated component has been proposed accordingly. It can provide both functions of differential-mode (DM) passband selectivity and commonmode (CM) rejection has been receiving increased attention. Over the past few years, much effort has been made in the exploration of various DM BPFs [1-9].

In [1], a DM BPF is proposed by adopting lumpedelement loaded half-wavelength resonators. In the design, the common-mode suppression can be tuned independently of the differential-mode response in the differential-mode filter via making full use of a center-loaded half-wavelength resonator. Although the DM BPF exhibits sharp frequency selectivity and high CM suppression, the involved design configuration and procedure are very complicated. Besides, another
DM BPF based on short-ended self-coupled ring resonator is presented in [2]. By periodically and symmetrically arranging short-ended SCRRs between the microstrip differential lines, the common-mode suppression can be realized at desired frequency easily with wideband differential-mode passband. But its circuit usually suffers from bulky size. In addition, by utilizing inherent common-mode rejection property of slotline, a compact DM BPF is developed in [3]. In this design, by properly placing the slotline resonator, the coupling between the slotline mode and the differential-mode signals can be maximized, while that between the slotline mode and the common-mode signals can be minimized. However, the employed slotline itself often results in a high insertion loss due to its high radiation loss. Moreover, based on the mode degeneration properties of the square patch resonator, a dual-mode differential mode has also been presented by adopting appropriate coupling structure among four isosceles right triangular patch resonators in [4]. But CM rejection level needs to be improved. On the other hand, stemming from the differential-mode coupled-line prototype, DM BPFs $[5,6]$ are proposed with improved performance by utilizing balanced coupled-line structure to realize the passband response in differential-mode operation and meanwhile give nice stopband in common-mode operation. Nevertheless, these designs are still difficult to achieve high and wide stopband rejection for DM and CM simultaneously. Apart 


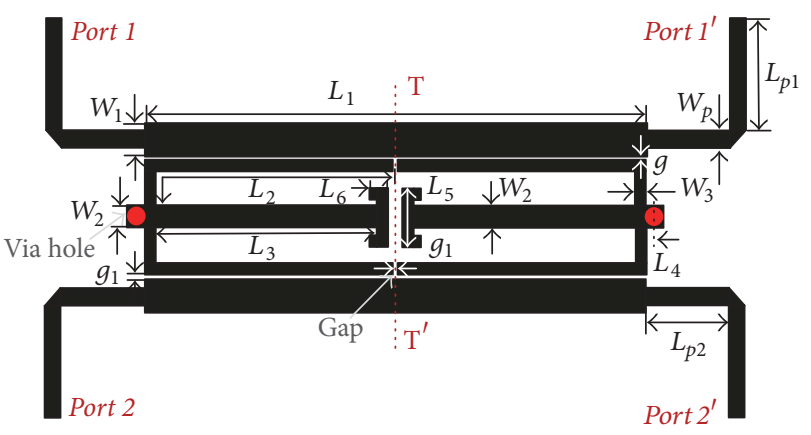

FIGURE 1: Schematic of the proposed differential-mode BPF.

from these above reported works, other differential-mode bandpass filters based on coplanar waveguide resonator, substrate integrated waveguide resonator, or dielectric resonator are also reported in [7-9].

In this letter, a microstrip differential-mode BPF is presented with new coupling topology by properly arranging multimode resonators between input/output transmission lines. High selective passband and wide stopband for DM and a wide and deep stopband for CM are realized at the same time in the design. For demonstration, a prototype differential-mode BPF is implemented. Both simulated and measured results are in good agreement, which validates the design concept.

\section{Proposed Differential-Mode BPF Design}

Figure 1 depicts the layout of the designed DM BPF, which only consists of a pair of $\lambda / 2$ microstrip transmission input/output lines and two triple-mode resonators. The entire structure is symmetrical with respect to the vertical central plane $\mathrm{T}-\mathrm{T}^{\prime}$. Under DM operation, the central plane $\mathrm{T}-\mathrm{T}^{\prime}$ behaves as a perfect electrical wall. On this occasion, parallel coupled line prototype with two different terminations of one open-ended circuit and one short-ended circuit will be formed up between the input/output lines and the arms of the resonators. It will provide a bandpass response. Therefore, the DM equivalent circuit of its half symmetrical bisections can be simply treated as a triple-mode bandpass filter with a pair of bandpass coupled feeding lines. Meanwhile, for common-mode operation, the central plane becomes a perfect magnetic wall. With this case, parallel coupled line prototype with two same short-ended terminations will be generated between the input/output lines and the arms of the resonators, which will exhibit an all stopband response. Correspondingly, the $\mathrm{CM}$ equivalent-half-circuit can be directly deemed as all-stopband circuit, since the feeding lines exhibit all-stop characteristic [10].

In order to illustrate the resonant properties of the employed resonator, Figure 2 describes the corresponding even-mode and odd-mode equivalent circuits of its half symmetrical bisections by utilizing the even-mode/odd-mode method in [11]. Therefore, assuming that the even-mode input admittance and odd-mode input admittance $Y_{\text {in,even }}$ and $Y_{\text {in,odd }}$, respectively, are equal to zero, the resonant condition equations can be derived as

$$
\begin{aligned}
\cot \theta_{3}-\tan \theta_{2} & =\frac{2}{K} \tan \theta_{1} \\
\theta_{1} & =\frac{\pi}{2}
\end{aligned}
$$

where $K=Y_{2} / Y_{1}$ is the admittance ratio of the resonator.

In addition, one inherent $\mathrm{TZ}$ can be found when $\theta_{2}=\pi / 2$ under $Y_{\text {in,odd }}=Y_{\text {in,even }}$. Based on the above analysis, the first three even-mode and odd-mode resonant frequencies can be deduced as $f_{\text {even } 1}, f_{\text {odd }}$, and $f_{\text {even } 2}$, respectively. Figure 3 shows the design chart of the employed triple-mode resonators with $K=1.6$. We can figure out that $f_{\text {even } 1} / f_{\text {odd }}$ and $f_{\text {even } 2} / f_{\text {odd }}$ are mainly, respectively, determined by $\theta_{3}$ and $\theta_{2}$, while $f_{\text {odd }}$ is only determined by $\theta_{1}$. More specially, it can be observed that $f_{\text {even } 1} / f_{\text {odd }}$ almost remains unchanged versus $\theta_{2}$ but decreases as $\theta_{3}$ increases.

Furthermore, $f_{\text {even } 2} / f_{\text {odd }}$ significantly decreases as $\theta_{2}$ decreases, while it causes a small reduction when $\theta_{3}$ rises up. Figure 4 describes the impedance ratio of $Y_{1} / Y_{2}$ influence on resonant frequencies and frequency bandwidth. It can be seen that when $Y_{1} / Y_{2}$ increases, $f_{\text {even } 1}$ and $f_{\text {odd }}$ fall down, while the bandwidth between $f_{\text {even } 1}$ and $f_{\text {even } 2}$ increases. Therefore, the impedance ratio of $Y_{1} / Y_{2}$ can be utilized to increase the design freedom. These above demonstrated properties are meaningful for one to realize a differential-mode BPF with desired DM passband bandwidth and high selectivity. Figure 5 gives the corresponding coupling scheme of the DM BPF under differential-mode operation, where nodes 1, 2, and 3 denote the even and odd modes of the resonator which form up the triple-mode DM filtering response, respectively. Due to the symmetry of the DM equivalent circuit, the coupling coefficients in Figure 5 satisfy the relationship of $M_{S, 1 / 2 / 3}=$ $M_{L, 1 / 2 / 3}$.

Herein, a prototype differential-mode BPF with the central frequency of $f_{0}=2.2 \mathrm{GHz}$ and desired $3 \mathrm{~dB}$ bandwidth of $240 \mathrm{MHz}$ is designed for an instance. The design procedure is listed in the following steps. At the beginning, according to the above (1a)-(1b), calculate the parameters $\left(L_{2}=20.4 \mathrm{~mm}\right.$, $L_{3}=18.0 \mathrm{~mm}$, and $L_{4}=0.8 \mathrm{~mm}$ ) of the resonator with the derived frequencies $f_{\text {even } 1}=2.11 \mathrm{GHz}, f_{\text {odd }}=2.24 \mathrm{GHz}$, and $f_{\text {even } 2}=2.33 \mathrm{GHz}$ from a targeted coupling matrix [12]. Secondly, based on the required external $Q$-factors to even and odd modes $\left(Q_{\text {exe1 }}=37.0, Q_{\text {exe2 }}=66.3\right.$, and $\left.Q_{\text {exo }}=23.8\right)$ from the matrix, determine the values of the width $\left(W_{1}=2.3 \mathrm{~mm}\right)$ and gap $(g=0.2 \mathrm{~mm})$ through extracting the two group delays, that is, $\tau_{S 11}\left(f_{\text {even }}\right)$ and $\tau_{S 11}\left(f_{\text {odd }}\right)$. In the end, the designed differential-mode circuit is initially built and fine tuning is performed to obtain optimal performance.

\section{Experimental Results}

To validate the design concept, the differential-mode filter has been designed and fabricated on the substrate with a relative dielectric constant of 3.55 , thickness of $0.508 \mathrm{~mm}$, and loss tangent of 0.0027 . The final layout parameters are $L_{1}=$ $35.6 \mathrm{~mm}, L_{2}=19.5 \mathrm{~mm}, L_{3}=17.1 \mathrm{~mm}, L_{4}=0.8 \mathrm{~mm}, L_{5}=$ 


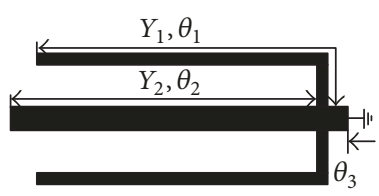

(a)

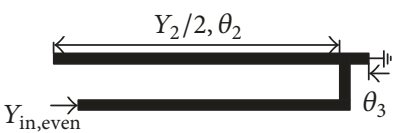

(b)

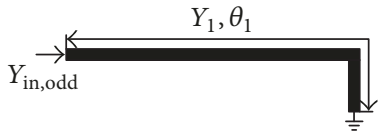

(c)

FIGURE 2: Equivalent circuits of employed multimode resonator. (a) Entire structure. (b) Even-mode bisection. (c) Odd-mode bisection.

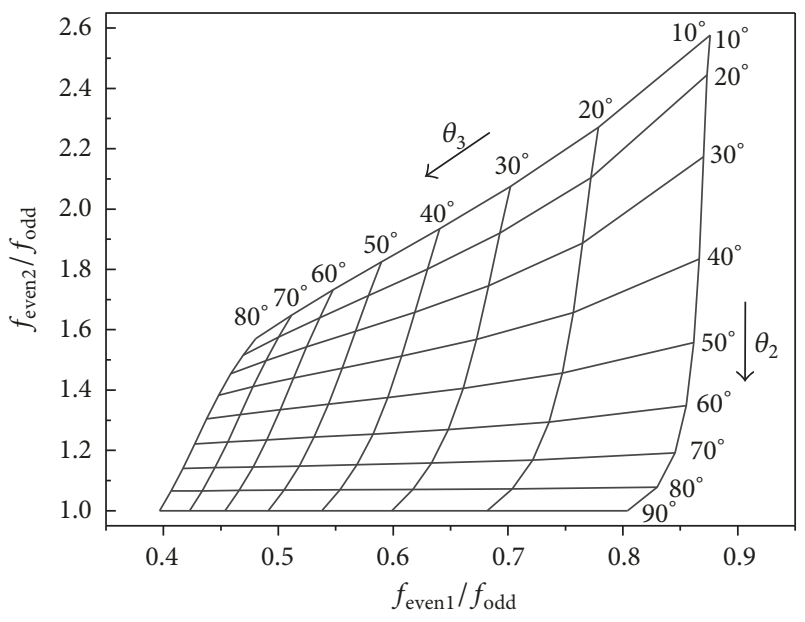

FIgURE 3: Design chart of the triple mode resonator.

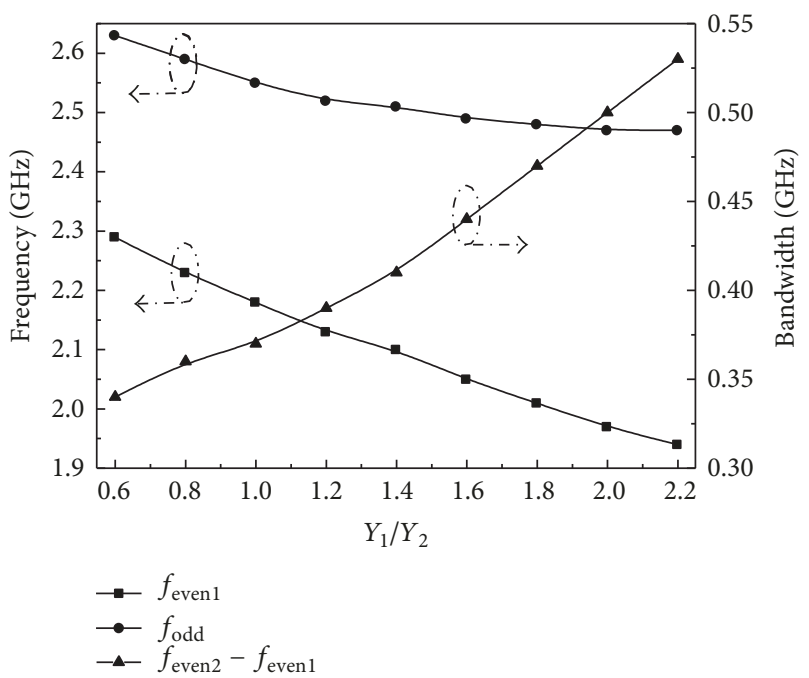

FiguRE 4: Resonant frequencies and bandwidth versus $Y_{1} / Y_{2}$.

$4.2 \mathrm{~mm}, L_{6}=1.3 \mathrm{~mm}, L_{p 1}=7.9 \mathrm{~mm}, L_{p 2}=6.0 \mathrm{~mm}, W_{1}=$ $2.3 \mathrm{~mm}, W_{2}=1.6 \mathrm{~mm}, W_{3}=0.7 \mathrm{~mm}, W_{p}=1.17 \mathrm{~mm}$, and $g=$ $0.2 \mathrm{~mm}$. The photograph of the fabricated circuit is displayed in Figure 6. Figure 7 shows the simulated and measured results. Simulation is accomplished by the commercial fullwave simulator ANSOFT HFSS, while the measurement is carried out in the Agilent N5244A four-port vector network analyzer. As shown in the figure, under DM operation, the measured DM central frequency is $2.2 \mathrm{GHz}$ with the $3 \mathrm{~dB}$ Bandwidth of $230 \mathrm{MHz}$. Three transmission poles inside the
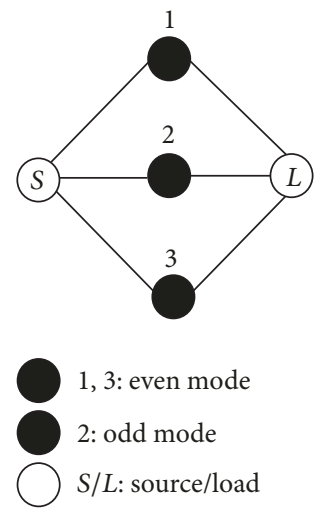

FIGURE 5: Equivalent-half-coupling scheme of the proposed DM BPF under differential-mode operation.

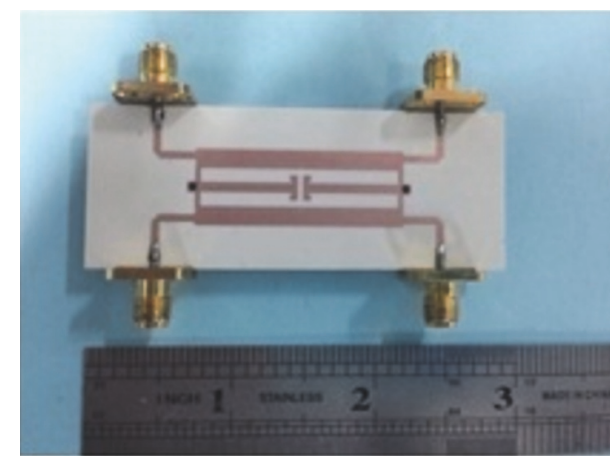

FIgURE 6: The photograph of the fabricated DM BPF.

passband can be clearly observed. Meanwhile, one transmission zero can also be obviously found as expected. Within this DM operating band, the measured minimum insertion loss (IL) is $0.8 \mathrm{~dB}$, while the return loss (RL) is better than $15.8 \mathrm{~dB}$. Besides, the proposed differential-mode BPF exhibits sharp frequency selectivity and good harmonic suppression with more than $30 \mathrm{~dB}$ suppression level up to $2.7 f_{0}$. On the other hand, for CM excitation, the differential-mode filter shows an ultrawide stopband with the rejection higher than $30 \mathrm{~dB}$ from DC to $2.8 f_{0}$.

Table 1 compares the performances of the proposed DM BPF with other published works in terms of the specifications, that is, roll-off, differential-mode stopband, and commonmode suppression. It indicates that our differential-mode filter exhibits not only sharp DM passband selectivity and wide upper stopband but also good ultrawide CM stopband rejection against others. 
TABLE 1: Comparisons with previous works.

\begin{tabular}{lcccc}
\hline Refs. & $\begin{array}{c}\text { Sharp } \\
\text { roll-off }\end{array}$ & $\begin{array}{c}\text { DM } \\
\text { suppression } \\
>30 \mathrm{~dB}\end{array}$ & $\begin{array}{c}\mathrm{CM} \\
\text { rejection } \\
>30 \mathrm{~dB}\end{array}$ & $\begin{array}{c}\mathrm{CM} \\
\text { rejection } \\
\left(0 \text { to } 2.5 f_{0}\right)\end{array}$ \\
\hline$[4]$ & Yes & None & None & None \\
{$[5]$} & Yes & Up to $2.0 f_{0}$ & Up to $1.9 f_{0}$ & $>26 \mathrm{~dB}$ \\
{$[6]$} & No & None & Up to $2.7 f_{0}$ & $>30 \mathrm{~dB}$ \\
{$[7]$} & Yes & Up to $2.3 f_{0}$ & Up to $1.3 f_{0}$ & $>10 \mathrm{~dB}$ \\
This work & Yes & Up to $2.7 f_{0}$ & Up to $2.8 f_{0}$ & $>31.4 \mathrm{~dB}$ \\
\hline
\end{tabular}

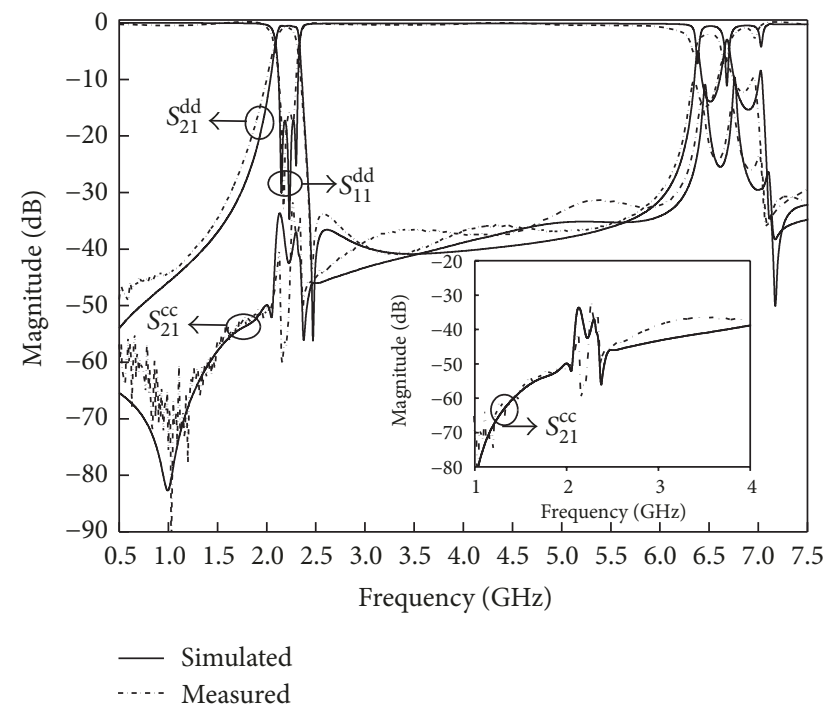

Figure 7: Measured and simulated results of the DM BPF.

\section{Conclusion}

In this letter, a compact differential-mode bandpass filter (BPF) has been presented with an ingenious coupling topology, achieving wide and deep stopband rejection for both differential mode and common mode. The explicit design procedure has been described and a prototype example has been manufactured. The experimental results agree well with simulated ones, validating the feasibility of the proposed design concept for modern differential communication system.

\section{Conflicts of Interest}

The authors declare that they have no conflicts of interest.

\section{References}

[1] J. Shi and Q. Xue, "Balanced bandpass filters using centerloaded half-wavelength resonators," IEEE Transactions on Microwave Theory and Techniques, vol. 58, no. 4, pp. 970-977, 2010.

[2] H. Wang, K.-W. Tam, S.-K. Ho, W. Kang, and W. Wu, "Shortended self-coupled ring resonator and its application for balanced filter design," IEEE Microwave and Wireless Components Letters, vol. 24, no. 5, pp. 312-314, 2014.

[3] D. Chen, H. Bu, L. Zhu, and C. Cheng, "A differential-mode wideband bandpass filter on slotline multi-mode resonator with controllable bandwidth," IEEE Microwave and Wireless Components Letters, vol. 25, no. 1, pp. 28-30, 2015.

[4] Q. Liu, J. Wang, and Y. He, "Compact balanced bandpass filter using isosceles right triangular patch resonator," IEEE Electronics Letters, vol. 53, no. 4, pp. 253-254, 2017.

[5] T. Yan, D. Lu, J. Wang, and X.-H. Tang, "High-Selectivity Balanced Bandpass Filter with Mixed Electric and Magnetic Coupling," IEEE Microwave and Wireless Components Letters, vol. 26, no. 6, pp. 398-400, 2016.

[6] D. Chen, L. Zhu, H. Bu, and C. Cheng, "Differential-mode bandpass filter on microstrip line with wideband commonmode suppression," IEEE Electronics Letters, vol. 53, no. 3, pp. 163-165, 2017.

[7] A. Fernández-Prieto, J. Bhatker, A. Lujambio, J. Martel, F. Medina, and R. R. Boix, "Balanced bandpass filter based on magnetically coupled coplanar waveguide folded-stepped impedance resonators," IEEE Electronics Letters, vol. 52, no. 14, pp. 1229-1231, 2016.

[8] K. Zhou, W. Kang, and W. Wu, "Compact dual-band balanced bandpass filter based on double-layer SIW structure," IEEE Electronics Letters, vol. 52, no. 18, pp. 1537-1539, 2016.

[9] J.-X. Chen, Y. Zhan, W. Qin, Z.-H. Bao, and Q. Xue, "Novel narrow-band balanced bandpass filter using rectangular dielectric resonator," IEEE Microwave and Wireless Components Letters, vol. 25, no. 5, pp. 289-291, 2015.

[10] L. Yang, W.-W. Choi, K.-W. Tam, and L. Zhu, "Balanced DualBand Bandpass Filter With Multiple Transmission Zeros Using Doubly Short-Ended Resonator Coupled Line," IEEE Transactions on Microwave Theory and Techniques, vol. 63, no. 7, pp. 2225-2232, 2015.

[11] D. M. Pozar, Microwave Engineering, Wiley, New York, NY, USA, 3rd edition, 2005.

[12] J. Hong and M. J. Lancaster, Microstrip Filters for RF/Microwave Applications, John Wiley \& Sons, New York, NY, USA, 2nd edition, 2001. 


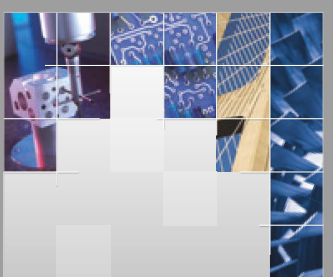

\section{Enfincering}
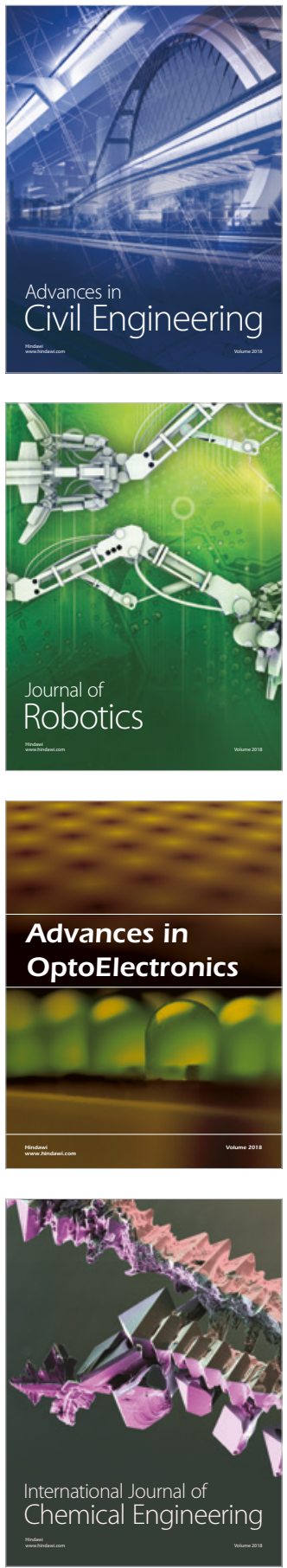

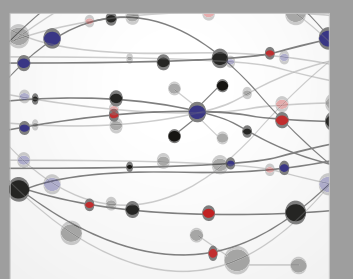

\section{Rotating \\ Machinery}

The Scientific World Journal

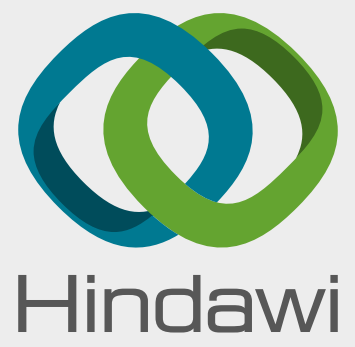

Submit your manuscripts at

www.hindawi.com
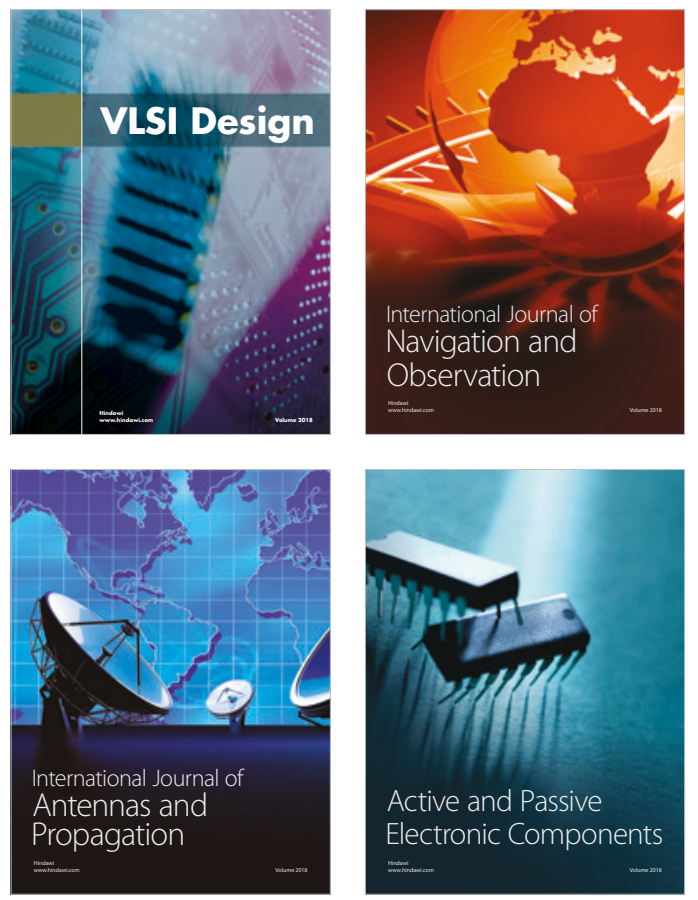
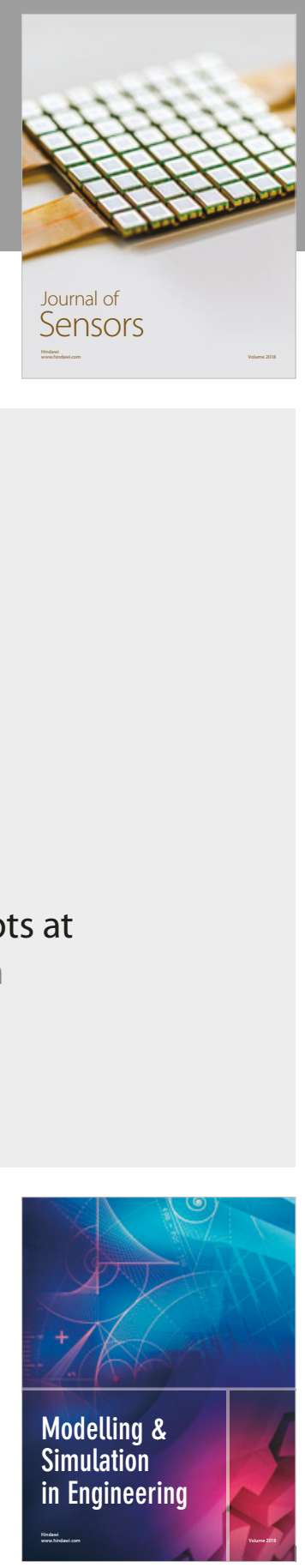

\section{Advances \\ Multimedia}
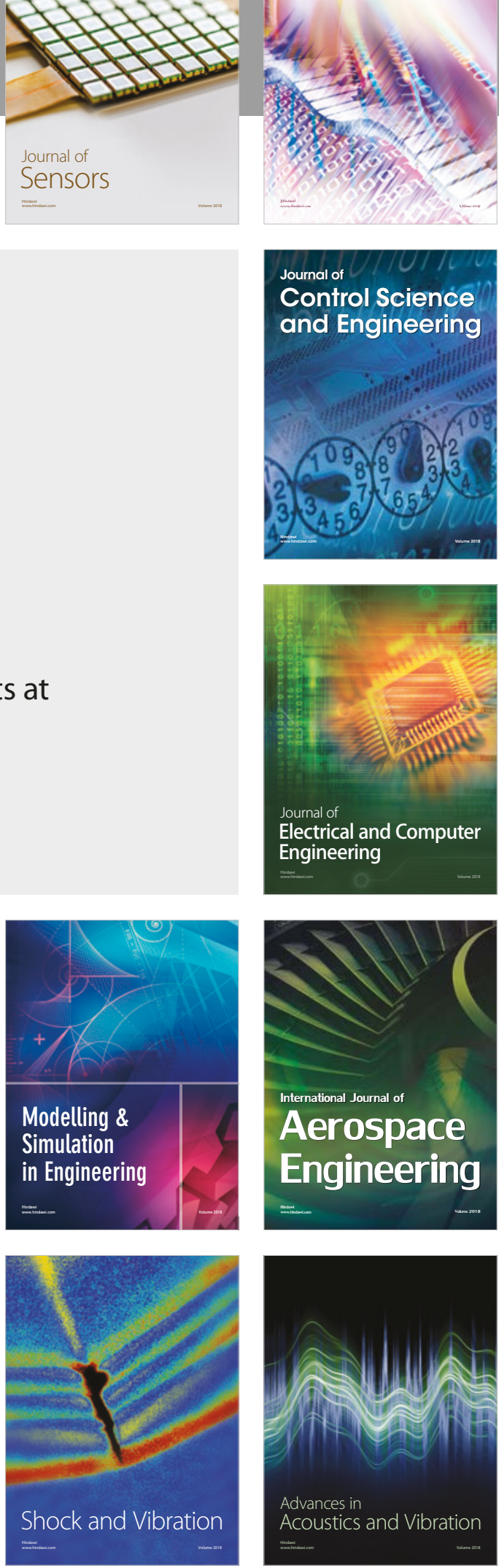